\title{
EL PARADIGMA DE LO COMÚN PARA LA TRANSICIÓN ECOSOCIAL: PRÁCTICAS DEMOCRÁTICAS PARA LA RECUPERACIÓN CIUDADANA DE LA ENERGÍA
}

\section{THE COMMON PARADIGM FOR ECOSOCIAL TRANSITION: DEMOCRATIC PRACTICES FOR CITIZEN ENERGY RECOVERY}

\author{
Ekhi Atutxa \\ Universidad del País Vaco \\ ekhi.atutxa@deusto.es \\ Imanol Zubero \\ Universidad del País Vasco \\ imanol.zubero@ehu.eus
}

\section{RESUMEN}

Ante la narración neoliberal imperante, el paradigma de lo común se convierte en una de las posibles maneras de auto-organización y mutuo reconocimiento de prácticas que ya muestran otras formas de hacer y de vivir en las diversas regiones del planeta. Lo común envuelve todas aquellas actividades (auto)transformativas producidas capaces de crear nuevas instituciones para la búsqueda de mayores cotas de autonomía humana y justicia social y ecológica. Desde la identificación de retos del sector energético español, este trabajo expone la contribución que el paradigma de lo común realiza desde la práctica colectiva y local a la transición ecosocial. Mediante la identificación de los criterios normativos y aplicados que caracterizan las prácticas comunes, se muestra una pequeña parte de la constelación de iniciativas que actualmente desarrollan su actividad en pro de la democratización del gobierno social y ecológicamente justo de la energía.

\section{PALABRAS CLAVE}

Suficiencia, Energía, Comunización, Praxis, Transformación, Encuentro.

CódIGos JEL: O10, O13.

Fecha de recepción: 18/07/2019

\section{ABSTRACT}

Facing the prevailing neoliberal narrative, the common paradigm is one of the possible ways of self-organization and mutual recognition of practices that already show other ways of doing and living in the various regions of the planet. The common involves all those (self) transformative activities capable of creating new institutions on the search for greater levels of human autonomy and social and ecological justice. Based on the Spanish energy sector challenges, this work exposes the contribution that the common paradigm makes from collective and local practices to the eco-social transition. By identifying the normative and applied criteria that characterize the common paradigm, a small part of the constellation of initiatives that currently develop their activity for the democratization of a socially and ecologically fair government of energy is shown.

\section{KEYWORDS}

Sufficiency, Energy, Commoning, Praxis, Transformation, Encounter.

Fecha de aceptación: 11/11/2019 



\section{ANTE LA TEOLOGÍA NEOLIBERAL}

Desde nuestra incorporación al capitalismo, se han consolidado los tres pilares de lo que R. Petrella (2009) denomina como narración imperante, 1) la fe en la tecnología, que a través de incesantes innovaciones, cada vez más revolucionarias, darían respuesta a problemáticas como las generadas por el cambio climático, 2) la confianza en el capitalismo y su capacidad de mutación, que incluso mediante la mercantilización de la propia vida a escala global, nos dirige hacía una sociedad del conocimiento cada vez menos dependiente de los recursos naturales y que permitiría el perpetuo crecimiento económico requerido por el sistema, y, 3) mediante aportaciones como El fin de la historia y el último hombre realizadas de F. Fukuyama en 1992, la convicción colectiva de la inexistencia de alternativas al sistema actual.

Estas creencias, han contribuido a la generación de una determinada y concreta relación de los seres humanos con aquello que nos rodea; nos consideramos superiores a cualquier otro elemento constitutivo de la naturaleza. No somos parte de la misma. Todo se ha convertido en recursos que podemos emplear, manipular, y explotar en pos del progreso. El sistema socioeconómico capitalista somete al sistema natural.

Durante años se han promovido numerosas propuestas, como el capitalismo verde que, en gran medida y en la jerga economicista, pretenden resolver las "externalidades" generadas por las diversas actividades económicas a través de soluciones de mercado y del desarrollo de nuevas tecnologías más sofisticadas y eficientes. El Protocolo de Kyoto sobre el cambio climático', con la implantación de mecanismos basados en el mercado como el comercio de los derechos de emisión (más conocido como el mercado del carbono) o los dirigidos a impulsar un desarrollo

1 El Protocolo de Kyoto, impulsado por la Naciones Unidades y adoptado en 1997 con el objetivo reducir las emisiones de gases que producen efecto invernadero, fue un fracaso desde el punto de vista de aceptación por parte de los países. Más información sobre el protocolo en Naciones Unidas (1998). Protocolo de Kyoto de la Convención Marco de las Naciones Unidas sobre el Cambio Climático. http://unfccc.int/resource/docs/convkp/kpspan.pdf. Con la firma del acuerdo por parte de 174 paises y la Unión Europea, COP21 en cambio parece haber sido un éxito en este sentido (http://newsroom.unfccc.int/es/acuerdo-de-paris/175-estadosfirman-el-acuerdo-de-paris/). 
limpio, es una muestra de ello². Nuestra supuesta condición de superioridad sobre la naturaleza, nos hace creer que, a través del mercado y la revolución tecnológica, seremos capaces de resolver cualquier contratiempo, por muy complejo e inabarcable que éstos puedan parecer.

A lo largo del tiempo, los seres humanos han reflexionado sobre los cielos con reverencia y fascinación. Es preocupante reconocer que nuestra era siempre será conocida por forzar este sentido humano de admiración a la competición por el interés de estudiar la mecánica del clima y la química del cielo. Innegablemente, la era de la gestión de la atmósfera está sobre nosotros. Su origen constitucional se puede remontar a la aprobación del Protocolo de Kyoto y las negociaciones en curso que buscan su interpretación y operacionalización ${ }^{3}$ (Byrne et al, 2004: 430)

Sin embargo, ni las soluciones parcialmente consensuadas en el Protocolo de Kyoto o en el COP21, ni la consolidación de la mencionada narración dominante, han sido eficaces para la resolución de las profundas contradicciones existentes entre la naturaleza y el capital. Parece que el capital no puede cambiar su manera de mercantilizar la naturaleza y transformarla en derechos de propiedad. La transición es así ineludible, pero ¿cómo se producirá?

Conviene que, de un modo planificado, avancemos hacia un cambio de modelo y, por qué no, aprovecharlo para comprender que puede ser una oportunidad para dirigirnos hacia sociedades ecológicamente y socialmente más justas. De cualquier forma, si no tomamos esta posición, el agotamiento de los recursos, la contaminación y las insostenibles desigualdades sociales nos obligarán. "El Cambio Climático nos obliga a hacer -y con urgencia- lo que en cualquier caso había que hacer: cambiar el modelo de desarrollo y en particular el modelo energético hacia una mayor sostenibilidad en una economía descarbonizada" (FER, 2011: 3). Una

2 Forman parte de los mecanismos de Kyoto el 1) Comercio de los derechos de emisión, 2) el Mecanismo para un Desarrollo Limpio, 3) la Aplicación Conjunta, y 4) el Fondo de adaptación. Más información sobre estos mecanismos basados en el mercado en la página web oficial de Naciones Unidas para el Cambio Climático: http://unfccc.int/portal_espanol/ informacion_basica/protocolo_de_kyoto/organizacion/mecanismos/items/6219.php

3 Traducción propia de su idioma en la versión original, "Throughout the ages, human beings have reflected on the heavens with reverence and fascination. It is troubling to recognize that our era will forever be known for forcing the human sense of awe to compete with a studious interest in the mechanics of climate and the chemistry of the sky. Undeniably, though, the era of atmosphere management is upon us. Its constitutional origin can be traced to the approval The Kyoto Protocol and the ongoing negotiations that seek to interpret and operationalize it". 
crisis ecológica estructural que es, sin duda, una de las mayores amenazas a la que nos enfrentamos en este siglo.

\section{EL INTERREGNO Y LA POSIBILIDAD DE UNA TRANSICIÓN ECOSOCIAL} ¿Cómo terminará el capitalismo?, titulaba en 2016 W. Streeck sus ensayos sobre lo que consideraba un sistema en decadencia. Según este sociólogo alemán, la profunda crisis sistémica nos aproxima a límites difícilmente superables. Ante las crisis de endeudamiento, la crisis bancaria y financiera, la crisis de la democracia, la crisis ecológica y la crisis geopolítica sugería que no asumiéramos la responsabilidad de contestar a la pregunta de qué proponemos poner en su lugar. Afirmaba que la esperanza de que un sujeto revolucionario preparado para dar fin al capitalismo como época histórica en pos del progreso de la humanidad se estaba desvaneciendo tras la destrucción, en la revolución neoliberal global, de la acción colectiva y de la esperanza de recuperarla (Streeck, 2017: 77). Coincidía con Z. Bauman (2015) en señalar que lo que vendría inmediatamente después del capitalismo no sería el socialismo o algún otro orden social definido, sino un interregno duradero (Streeck, 2017: 29). Tal vez un interregno en el que ya nos encontramos pero que hasta que tomemos la distancia temporal suficiente no seamos capaces de reconocerlo.

Ciertamente, parecemos estar sumergidos en un momento en el que las instituciones sociales, políticas, económicas y culturales tradicionales se encuentran en entredicho (Geiselberger, 2017; Mishra 2017; Lilla, 2016). Asimismo, no cabe duda de que la neoliberalización del capitalismo ha perjudicado, más si cabe, nuestra capacidad de imaginar una posible alternativa sistémica. Sin embargo la acción colectiva no ha desaparecido (Alonso et al, 2017, Martínez, 2014; Bollier, 2003; Ostrom, 2000). No ha sido "totalmente destruida". Entre las fuertes marejadas que producen las constantes crisis que se originan en múltiples dimensiones, no sólo perviven y se mantienen, sino que emergen con fuerza e incesantemente iniciativas colectivas que, apoyadas en valores como la solidaridad y la reciprocidad, luchan por la consecución de una mayor justicia social y ecológica. De hecho, lo que resultará tras el duradero interregno dependerá en gran medida de la activación de iniciativas colectivas experimentales que puedan enfrentarse y mostrar que hay alternativa a las lógicas imperantes. En el interregno, en el "mientras tanto", la transición ecosocial debe pensarse como un proceso posible que requiere de la convicción y acción de cada vez un mayor número de personas.

Ante esta desasosegante, pero al mismo tiempo esperanzadora realidad, la idea de lo común se convierte en una de las posibles maneras de (auto) organización y mutuo reconocimiento de prácticas que ya muestran otras formas de hacer y de vivir en las diversas regiones del planeta. Lo común 
envuelve todas aquellas actividades (auto) transformativas producidas por sujetos que, mediante la acción conjunta, son capaces de crear nuevas instituciones en constante cambio para la búsqueda de mayores cotas de autonomía humana (Laval y Dardot, 2015). Una autonomía humana que nos pueda dirigir a escenarios de una mayor justicia social y ecológica; a la transición ecosocial.

\section{LO COMÚN: UN PARADIGMA QUE AGLUTINA, ENVUELVE Y RECUPERA}

Como alternativa a lo público-estatal y lo privado-mercantil, el paradigma de lo común cuenta con los elementos definitorios suficientes para analizar, desde una misma y transversal perspectiva, prácticas aparentemente lejanas que actúan en infinidad de ámbitos imaginables a favor de una mayor justicia social y ecológica. Posibilita el reconocimiento mutuo de prácticas heterogéneas que desde la acción colectiva y cooperativa local comparten un mismo horizonte en respuesta a problemáticas que adquieren una dimensión global.

Si las iniciativas de la Economía Social se han pensado ocasionalmente desde su capacidad de completar los espacios abandonados por las esferas de lo público y lo privado, lo común parece abrir la puerta a una mayor comprensión, a una toma de conciencia, sobre la posibilidad de la recuperación democrática de espacios privatizados mediante la activación de prácticas cooperativas y solidarias que actúan desde la lógica de la reciprocidad. Así, la comunización ${ }^{4}$ o el commoning, la conversión de diversas realidades en comunes, es un hecho (Byrne et al 2002, 10). Una alternativa que ya existe frente a los procesos de acumulación por desposesión descritos por D. Harvey (2005).

La comunización significa que las instituciones de lo común se pueden (re)producir (Rayner, 2012). Dan cuenta de ello experiencias de alto impacto de los últimos años como el movimiento occupy o el $15 \mathrm{M}$ en España. Fenómenos que fueron capaces de reclamar espacios públicos, gestionados desde la Administración Pública para convertirlos en bienes gobernados por la praxis común. Espacios que pasaron temporalmente a ser zonas autónomas para compartir, aprender y para la participación y transformación democrática.

En efecto, lo común contribuye a mostrar desde la praxis que las categorías de lo público, lo privado y lo social son hechos sociales. La comprensión de que nos encontramos ante categorías socialmente construidas nos lleva a reconocer su maleabilidad. Es posible que las instituciones públicas

4 Comunización también conocido como Commoning en inglés, es un término popularizado por P. Linebaugh en 2008, en El Manifiesto de la Carta Magna. Comunes y libertades para el pueblo. 
tradicionales incorporen características de los comunes haciéndose más democráticas y participativas, del mismo modo en el que es posible que las entidades con formas jurídicas como las Sociedades Limitadas ubicadas tradicionalmente en la esfera de lo privado prioricen los fines sociales en contraposición a la consecución de una mayor rentabilidad económica. Un ejemplo clarificador de esto último es la iniciativa Ecooo, una empresa de no lucro fundada en 2005 y centrada en todo tipo de proyectos que activen a la ciudadanía a favor de un nuevo modelo energético, limpio, sostenible y en manos de las personas.

Lo común se caracteriza por su potencial transversalizador. Iniciativas de características similares, a priori ubicadas en ámbitos de muy diversa índole, que son capaces de reconocerse en una empresa común. La búsqueda de una mayor autonomía humana y una mayor justicia social y ecológica.Los comunes emergen constantemente y se afianzan en todo ámbito imaginable. De hecho, desde las Tecnologías de la Información y la Comunicación (TICs) con iniciativas como Wikipedia hasta los bosques comunales de Galicia, la praxis instituyente común en su diversidad y extensión resulta inabarcable. Cualquier bien puede ser gobernado desde lo común. De este modo, a pesar de las rígidas instituciones jurídicas que hoy dan forma a su producción, gestión, transformación, distribución y consumo, también un bien social como la energía es "comunizable".

Un bien que es, de hecho, un pilar fundamental para la construcción de las sociedades actuales y nuestras instituciones sociales, políticas, económicas y culturales (Mitchel, 2011).Un bien social que ciertamente, hoy en día, es gobernando a través de un modelo energético social y ecológicamente injusto. Un bien que, por su relevancia social, requiere un gobierno democrático que el mercado y el Estado en sus formas actuales no consiguen proveer.

\section{LA ENERGÍA COMO BIEN SOCIAL COLECTIVAMENTE GENERADO: LA ENERGÍA EN COMÚN}

La energía siempre ha estado vinculada, no sólo a la supervivencia del ser humano, sino también a la propia reproducción social de la vida (Riutort, 2017: 83). La función que la energía juega en la sociedad es decisiva. No sólo porque es necesaria para la cobertura de las necesidades básicas, sino también porque es un pilar fundamental de las instituciones sociales colectivamente conformadas como es el caso de la propia democracia.

De hecho, podríamos afirmar que el modelo energético actualmente imperante es parte de las raíces del sistema capitalista que se ha consolidado con el paso del tiempo. Totalmente necesario para la pervivencia y reproducción de los hábitos y las formas de vida que nuestras sociedades 
mayoritariamente defienden y promueven. Además del capitalismo, la misma democracia ha sido moldeada por el modelo energético.

Sin la energía que obtienen del petróleo, sus formas actuales de vida política y económica no existirían. Sus ciudadanos han desarrollado formas de comer, viajar, alojarse y consumir otros bienes y servicios que requieren grandes cantidades de energía proveniente del petróleo y otros combustibles fósiles. ${ }^{5}($ Mitchell, 2011: 6).

La energía se encuentra tan en la raíz que se ha convertido en un factor indispensable para la comprensión de cualquier ámbito de la vida (además del económico, el político, el social, el ambiental, etc.) y las interacciones que se producen entre ellos. Así, la energía y el modelo alternativo que está aún por construir, tendrían que pensarse como palancas del bienestar de las comunidades locales y de sus actividades productivas y reproductivas cotidianas. También como dispositivo para la atenuación de las situaciones de vulnerabilidad y pobreza. Reconocer el carácter social de la energía y, en consecuencia, comprender la imperante necesidad de un gobierno democrático de la misma es uno de los primeros pasos a dar para una transición energética ecosocial.

Es determinante tomar la dirección hacia una profunda transformación en el modo en que se produce y consume energía, dando respuesta a las necesidades energéticas de todas las personas que habitamos el planeta a través de una relación armoniosa con el medioambiente. Para ello, es imprescindible avanzar hacia un gobierno democrático de la energía.

La ciudadanía tendría que estar en el centro de la producción, transporte, distribución y consumo de cualquier tipo de bien, pero más si cabe en el caso de bienes sociales como la energía. Para ello, no nos queda otra opción que transformar profundamente el modelo energético tradicional. No es suficiente con cuestionar el sistema capitalista, hay que poner en entredicho el sistema de valores que sostiene el modelo de desarrollo occidental (Urkidi et al, 2015: 211). Sin dañar el medioambiente y al resto de personas, para que la humanidad en su totalidad pueda disponer de energía es ineludible abandonar los combustibles fósiles, socializar y democratizar los medios de producción y transformar el modelo de consumo hasta la consecución de un estado de suficiencia.

5 Traducción propia de la versión en su idioma original, "Without the energy they derive from oil their current forms of political and economic life would not exist. Their citizens have developed ways of eating, travelling, housing themselves and consuming other goods and services that require very large amounts of energy from oil and other fossil fuels". 


\section{LA TRANSFORMACIÓN DEL MODELO ENERGÉTICO DESDE LA PRAXIS COMÚN}

El modelo energético globalizado y sus social y ecológicamente injustas características como 1) los insostenibles niveles de consumo y producción de los países industrializados y la consecuente deuda ecológica contraída por los mismos (Martínez-Alier y Oliveras, 2003), 2) el profundo déficit democrático del gobierno de la energía, y la alta concentración del poder económico y político (Gomez, 2004, Martil, 2016), 3) la contribución a la profundización de las estructurales situaciones de pobreza desde su dimensión energética (Tirado et al, 2014) y 4) la contaminación, la sobreexplotación de los recursos naturales y la huella ecológica (National Footprint Accounts, 2019), no pueden ser resueltas a través de una mayor penetración de la fuerza de mercado ni a través de los venerados avances tecnológicos.

El paradigma de lo común tiene el potencial de atenuar e incluso resolver algunas de estas estructurales problemáticas. Es capaz de reconocer la energía como un bien social y no como una mera mercancía de la que extraer las mayores rentas posibles. No sólo eso, tiene el potencial de contribuir a un gobierno democrático de la energía a través de la socialización de los medios de producción.

Los comunes cuestionan en profundidad las convicciones y creencias sobre las más óptimas fórmulas de gestión de todo tipo bienes, mostrando que hay una infinidad de maneras de organizarse socialmente, no sólo la del mercado o la del Estado. Los comunes son "(...) realidades institucionales que mantienen relaciones complejas -no necesariamente enfrentadascon otras instituciones económicas y sociales (...)" (Rendueles, 2017: 54). Son justamente estas relaciones complejas las que sirven también para controvertir desde la praxis, la sola existencia de tres ámbitos separados y únicos de lo público, lo privado y lo social y de influir en sus finalidades, modelos de gobierno y valores.

Frente a la gran incertidumbre sobre la evolución que tendrán las instituciones actuales, lo común es ya un conglomerado de prácticas instituyentes válido para producir transformaciones en los espacios de lo público, lo privado y lo social. En el ámbito energético, las 1) Las Ecoaldeas como Lakabe o Arterra Bizimodureferentes del neoruralismo, la ocupación rural, la vida en comunidad y la vida ecológica.2) Las Cooperativas de energía renovable como Goiener, Som Energia o Solabria o empresas sociales como Ecooo para la gestión y, gradualmente, para la generación ecológica de la energía. Entidades que fomentan el activísimo energético de la ciudadanía también a través de la pedagogía y concienciación en cuanto el consumo energético.3) Los partenariados público-ciudadanos como Rubí Brilla para la consecución de una mayor eficiencia energética 
y el uso de energías renovables en entornos industriales, comerciales y domésticos ${ }^{6}$. 4) Los colectivos de generación de conocimiento y de investigación-acción alternativos como el Observatorio Crítico de la Energía o el Instituto de Transición Rompe el Círculo son sólo un ejemplo de ello. El cuestionamiento, por parte de los comunes, de los espacios e instituciones socialmente construidos, propicia la inoculación de los valores como la solidaridad global, la justicia social y ecológica, y la equidad al resto de las esferas.

El siguiente decálogo pretende mostrar y visibilizar las potencialidades de la praxis instituyente común para abordar una transición energética ecosocial. Puntos de luz que alimentar para que desde lo local se pueda escalar hasta las acuciantes soluciones globales.

\subsection{SUFICIENCIA Y LA TOMA DE CONCIENCIA DE LAS}

\section{FRONTERAS DE POSIBILIDADES DE PRODUCCIÓN (Y CONSUMO)}

Sólo quien se autolimita deja existir al otro (Riechmann, 2004). Los avances y mejoras tecnológicas y organizativas, no supondrán un beneficio ecológico si no se pone en práctica el principio de la autocontención. Señala que, para avanzar hacia la sustentabilidad, el ser humano debe autolimitar sus capacidades en seis ámbitos clave, entre los cuales se encuentra el uso de la energía exosomática ${ }^{8}$ y la apropiación de recursos naturales (Riechmann, 2004: 152-155). Según este autor, la "obsesión productivista" de nuestras sociedades es la que se encuentra detrás de la crisis ecológica y para dar una respuesta eficaz a la misma, es necesario que, desde lo racional pero también desde lo afectivo, lo sensitivo y lo emocional, pasemos de la producción de mercancías a la producción y reproducción de vida.

6 Es tal la relevancia que este tipo de iniciativas han adquirido en los últimos años que en 2016 se propone una nueva Directiva del Parlamento Europeo y del Consejo sobre normas comunes para el mercado interior de la electricidad haciendo mención expresa a Las comunidades energéticas locales orientadas a la generación de valor en términos sociales, económicos y medioambientales más que a la rentabilidad financiera (Consejo de la Unión Europea, 2019: 65), posibilitando una mayor participación de los consumidores en la toma de decisiones del mercado energético a través de la generación distribuida.

7 Frontera de posibilidades de producción es un concepto recurrentemente empleado y fuertemente consolidado en la economía clásica que refleja las cantidades máximas de bienes y servicios que una sociedad es capaz de producir en un determinado período y a partir de unos factores de producción y unos conocimientos tecnológicos dados. Llama la atención que nunca se haya planteado una Frontera de posibilidades de consumo. Parece que el nivel de consumo este condicionado solamente por el nivel de producción e importaciones posible en una economía. La ortodoxia siempre ha considerado el aumento del consumo como algo positivo para la activación y sostenimiento de una economía.

8 Entendemos por energía exosomática por aquella energía externa generada fuera del cuerpo humano, que no proviene de su metabolismo y que es empleada para el desarrollo de todo tipo de actividades imaginables. La energía exosomática es la que nos provee de luz, calor, frio, etc. y posibilita actividades tan fundamentales como el suministro de agua, el transporte, cualquier tipo de industria, etc. 
No envano, J.Riechmannhace referencia directa a lasnuevas comunidades amigas de la tierra regidas por principios de mesura y cordura, denominadas como tales por M. Sacristán (2003: 275). Comunidades que revalorizan la pequeña escala siendo capaces de yuxtaponer a la racionalidad técnica una racionalidad de los valores. Una reorganización social que dejará a un lado la maximización del beneficio privado en pos de la autorregulación, una situación de estabilidad y equilibrio dinámico (Riechmann, 2004: 163-165). No es descabellado asimilar la praxis instituyente común a esas comunidades imaginadas por Sacristán. Los comunes no son otra cosa que la creación de nuevas instituciones locales para la consecución de esa racionalidad de los valores basada en los principios de la mesura, la cordura y la autolimitación.

En su dimensión normativa como la búsqueda de autonomía humana o la defensa de la justicia social y ecológica, los comunes son prácticas que intrínsecamente contribuyen a esta idea de la suficiencia y autocontención. Al tratarse de prácticas locales estrechamente conectadas al territorio y que gobiernan bienes utilizados por comunidades concretas, los comunes permiten la comprensión y la interiorización de la existencia de límites. Tanto desde el punto de vista de la producción como del consumo, una comunidad local se encuentra con límites físicos ineludibles cuando se enfrenta a la gestión de recursos limitados como la energía. Incluso en los casos de la solar, la eólica o la marítima ya que, aunque a largo plazo sean fuentes de energía inagotables, su producción diaria es limitada.

Cuando una comunidad en el gobierno de bienes como la energía, opera a escala local, es decir, produce, transporta, distribuye y consume en el lugar en el que desarrolla su cotidianidad, no tiene otra opción que tomar conciencia de sus verdaderas fronteras de posibilidades de producción (y de consumo). Fronteras que inevitablemente se diluyen al aumentar la escala y perder el nexo con el espacio físico y social en el que se (re) producen, en última instancia, las comunidades.

\subsection{UNA OPCIÓN POLITICA PARA LA TRANSICIÓN ECOSOCIAL}

Las nuevas alternativas energéticas, así como el propio cambio climático, son un nuevo tipo de objetos tan políticos como científicos, en el que confluyen una gran diversidad de disciplinas (Lafuente, 2007: 171). Añadiría que incluso más políticos que científicos. La transición a sociedades social y ecológicamente más justas es siempre una opción política y frente a este hecho, la praxis instituyente común puede jugar un papel decisivo. Para que estos cambios se produzcan, resulta crítico crear nuevas instituciones sociales en cuyo proceso las prácticas participativas locales tienen el potencial, no sólo de incluir a la ciudadanía en la toma de decisiones y en 
la reapropiación de los medios de producción, sino también de que éstas últimas estén embebidas de una ética secular común.

La praxis instituyente común mediante su gobierno participativo y democrático sitúa en manos de las propias comunidades la capacidad de decidir el modelo energético que más les conviene. Pero no se limitan a distribuir el poder entre los miembros de la comunidad. Lo común en su dimensión normativa se diferencia de otras prácticas colectivas por la búsqueda de la autonomía humana y de la justicia social y ecológica a través de la reciprocidad y la co-actividad ${ }^{9}$. La generalización de estas prácticas, mediante formulaciones democráticas de la actividad colectiva, sin ninguna duda, mermaría la actual concentración de poder económico y político, favoreciendo comportamientos sustentables eficaces para la resolución de la crisis económica, social y medioambiental a la que ineludiblemente debemos hacer frente.

\subsection{SOCIALIZACIÓN DE LOS MEDIOS DE PRODUCCIÓN}

Si bien es cierto que la aparición de tecnologías que favorecen la generación y consumo distribuido de energía ${ }^{10}$ posibilita la ubicación de la ciudadanía en el centro del sistema, sería asimismo ingenuo pensar que las grandes compañías energéticas no pretenderán continuar dominando el sector desde las lógicas mercantiles y el ánimo de lucro. Aun cuando el sector pueda estar tecnológicamente descentralizado. La descentralización no supone automáticamente la reapropiación de un bien básico como la energía por parte de la ciudadanía.

En esta pugna sobre quién se apropiará y dominará el sistema descentralizado, el paradigma de lo común podría volver a jugar un papel determinante. Lo común, en su dimensión normativa, facilita la toma de conciencia de bienes sociales colectivamente creados y, por ende,

9 Se debe producir la convergencia de todos los elementos mencionados. No se trata únicamente de distribuir el poder en los miembros de una determinada comunidad. La propia comunidad debe desarrollar su actividad orientada a una transición ecosocial posible para toda la humanidad. Las iniciativas del ámbito energético anteriormente mencionadas responden a esta necesaria convergencia. Tanto las Cooperativas de Energía Renovables como los partenariados público-ciudadanos o las Comunidades energética locales no sólo pretenden devolver el poder a la ciudadanía. Al mismo tiempo pretenden transitan a modelos socialmente más equitativos y ecológicamente más sostenibles.

10 Energía distribuida, generación descentralizada, generación dispersa, generación in-situ o generación embebida son novedosas aproximaciones, cada una con sus matices provenientes de los múltiples factores que intervienen en la definición, pero que al mismo tiempo tienen elementos en común que podrían resumirse en la producción de energía a pequeña escala, cerca del consumidor y que puede ser conectado al propio usuario o a la red de transporte y distribución (Fenercom, 2007: 9-10).La energía se generaría así en un gran número de nodos repartidos por toda la geografía, los cuales serían además capaces, no sólo de producir la energía necesaria para una comunidad, sino que también de revertir a la red eléctrica en caso de excedente y proveer así a otras comunidades. 
que deberían ser gobernados por todos/as. La comunización requiere de la participación hombro con hombro de todos los integrantes de la comunidad, no habiendo sociedad que no se haya sustentado en la gestión comunal de bienes (Linebaugh, 2010). No solamente porque todos/as (también con la contribución de las generaciones precedentes a través de los conocimientos acumulados con el paso del tiempo), hemos participado en la creación y construcción de estos bienes, sino porque éstos son el mismísimo sustento de nuestras sociedades.

En su dimensión aplicada, los comunes también podrían condicionar el uso social que se realizaría de las oportunidades que ofrece la tecnología necesaria para una generación distribuida. El cambio tecnológico puede contribuir y/o posibilitar un cambio social positivo, en el que haya una mayor justicia social y ecológica, pero no es suficiente. Los avances tecnológicos deben siempre ir acompañados de instituciones sociales capaces de hacer que sus frutos beneficien a todos los miembros de una sociedad y que al mismo tiempo desde la eficiencia y la suficiencia conlleve un uso sostenible de los recursos requeridos.

Si las prácticas instituyentes comunes se caracterizan por su gobierno democrático e inclusivo, que en la búsqueda de una mayor autonomía humana evitan adentrarse en las lógicas mercantiles, podemos afirmar que, junto a otras propuestas como la democracia energética o la economía social y solidaria, contribuyen a que la ciudadanía sea la que en última instancia ocupe el espacio central en el gobierno de la energía.

\subsection{COMUNIDADES LOCALES EN SITUACIONES DE VULNERABILIDAD}

Como consecuencia directa del modelo de desarrollo imperante se encuentra de forma transversal la desigualdad. La generalización del modelo de la historia económica moderna en forma de una serie secuencial de etapas de crecimiento económico (Rostow, 1961) ha demostrado ser errónea o insuficiente en el mejor de los casos. Insuficiencia que queda constatada por 1) La no consideración de las interacciones de los procesos económicos y sociales, 2) las relaciones de dependencia a escala regional, nacional e internacional, así como 3) la reducción del concepto de bienestar a la riqueza económica de cada país sin profundizar en cuestiones como la distribución o el acceso a la producción entre otras, ha imposibilitado una solución a las desigualdades que se producen tanto dentro de cada Estado-nación como en términos planetarios del Norte-Sur.

Un sistema capitalista desregulado y fortalecido por el proceso globalizador, impulsado por organismos como la Organización Mundial del Comercio, está impidiendo políticas locales encaminadas a promover un modelo energético alternativo (Klein, 2015: 56-69). Una escala de intervención local considerada clave para posibilitar una respuesta 
inmediata a las situaciones de urgencia que sufren los colectivos más desfavorecidos. La escala municipal o niveles incluso menores son propicios para actuar frente a situaciones de pobreza. Por su idoneidad para concretar metodologías, integradas a las existentes en intervención social, para la detección de la pobreza en su expresión energética o para realizar las actuaciones adaptadas a la idiosincrasia de cada lugar (Tirado et al, 2014: 137). La escala local, desde la perspectiva de lo común adquiere una mayor relevancia si cabe.

La praxis común, en tanto que actividad que se desarrolla por parte de comunidades locales, es la institución de la fuerza colectiva. Un conjunto de personas, mediante la práctica colectiva, produciría nuevas reglas independientes a las establecidas y en ocasiones en contraposición a las mismas para la consecución de mayores cotas de autonomía humana (Laval y Dardot, 2015: 460). Siendo el fin de las comunidades comunes incrementar la autonomía humana de sus miembros, inevitablemente desarrollarían una praxis autotransformativa en el que se buscaría la autonomía para todos ya que "su realización no puede concebirse plenamente más que como empresa colectiva" (Castoriadis, 2007:170). Las situaciones de pobreza en sus múltiples expresiones no tendrían cabida en las prácticas comunes.

\subsection{AUTONOMÍA DESDE LA CONEXIÓN}

La autonomía no quiere, en ningún caso, decir desconexión con otras prácticas similares, pero al mismo tiempo, heterogéneas que también abogan por sociedades ecológicamente y socialmente más justas. Todo lo contrario, los comunes no sólo son prácticas que promueven la cooperación entre los miembros de la comunidad. Esta cooperación debe salir de la comunidad y extenderse a otras prácticas, tanto del mismo ámbito como de otros que estén avanzando hacía el mismo horizonte.

Estas prácticas locales, conscientes de su pertenencia a un sistemamundo (Ramonet, 2011), en la búsqueda una mayor justicia social y ecológica, también tendrían el potencial de permitir y promover la autonomía humana de las personas que no formarían parte activa de la comunidad. Desde los comunes, no se quiere la autonomía solamente para los miembros de la comunidad sino para todas las personas que habitamos el planeta. Independientemente del objetivo de la práctica, la centralidad de la ciudadanía en la construcción de las soluciones a las diversas necesidades se convierte así en uno de los elementos transversales (Pradel et al, 2018: 193). Estaríamos ante una comunidad de comunidades que interactuarían desde la reciprocidad y no desde el ánimo de lucro y el intercambio mercantil.

De hecho, en la práctica los comunes de la energía colaboran con otras iniciativas del mismo ámbito, y también con experiencias que están 
emergiendo en entornos tan dispares como el financiero con la presencia de la banca ética o el de las TICs que defienden cuestiones como la apertura y neutralidad de internet. Mediante la conjunción de las tecnologías distribuidas actualmente existentes y la praxis instituyente común, sería posible que desde las comunidades locales se gestionara una energía que, a su vez, podría estar interconectada para aprovechar de manera reticular la renovables existentes en distintas regiones y así reducir la dependencia de recursos energéticos no renovables provenientes de lugares situados a miles de kilómetros de distancia.

\subsection{DECRECIMIENTO PARA UNA VIDA DIGNA}

Si pensamos el decrecimiento en los términos en los que lo hace $C$. Taibo, es decir, si entendemos que el decrecimiento forma parte de los movimientos que pretenden ofrecer una alternativa al sistema capitalista desde las prácticas colectivas y autogestionarias capaces de cancelar la ilusión del crecimiento indiscriminado (Taibo, 2014: 75-101), no nos resultará complejo resituarnos en el paradigma de los comunes.

Respetando los matices propios de la heterogeneidad de aproximaciones existentes para dar respuesta a los acuciantes problemas globales a los que tenemos que enfrentarnos como humanidad, en la idea de los comunes encontramos el punto de unión de múltiples y diversas iniciativas y acciones que desde campos de lo más variopintos contribuyen a una mayor justicia social y ecológica.

El simple hecho de que, desde la pluralidad, la enorme constelación de prácticas colectivas alternativas pueda encontrarse y reconocerse en el paradigma de lo común es en sí mismo una significativa contribución. Siguiendo la analogía de J. Holloway, desde las acciones teórico-prácticas diarias de rechazo y cuestionamiento del sistema se deben buscar y/o generar las grietas al sistema (Holloway, 2011: 8-10). Lo común, tanto desde lo normativo como desde su dimensión aplicada, asiste en esta doble tarea. Nos dota de nuevas narrativas alternativas a las hegemónicas y convencionales; es capaz de generar nuevas instituciones sociales que desde la praxis nos muestran distintas y democráticas formas de organización social; y nos hace tomar conciencia de los incesantes procesos de cercamiento que sufrimos en espacios tan dispares como el internet o tan inseparables de nosotros como son nuestros propios cuerpos.

\subsection{DESCENTRALIZACIÓN DEL PODER}

La legislación estatal del sector energético español, impide o, en el mejor de los casos, obstaculiza el florecimiento de iniciativas colectivas para el gobierno democrático de un bien como la energía. Trabas que tienen su origen en los poderes económicos afianzados en el sector privado 
que, con la intermediación de lo público (Huerta, 2005: 121-150), no permiten la exploración y menos aún la generalización de nuevos modelos energéticos descentralizados, locales y ciudadanos. Las protestas por el déficit democrático a la hora de diseñar los recientes marcos regulatorios del sistema eléctrico son innumerables (FER, 2013; García y Martín, 2014). Hasta el momento, el poder de decisión sobre cómo debería ser la configuración del sistema energético español nunca ha estado al alcance de la ciudadanía.

Al igual que ha sucedido en otras experiencias socialmente innovadoras (Pradel et al, 2018: 193), es necesaria una descentralización del poder en la que una ciudadanía, ubicada en el centro de un modelo alternativo, tenga la capacidad de decidir democráticamente el presente y el futuro de la generación y provisión energética. No obstante, la reubicación del poder de decisión en manos de la ciudadanía no es suficiente para caminar hacia un modelo energético social y ecológicamente más justo a nivel global. Un modelo actualmente imaginado, que dotaría de una mayor autonomía humana a las diversas comunidades locales del planeta sin reducir la de las demás, no requiere solamente de una mayor democracia.

La autogestión colectiva y democrática debe ir acompañada de nuevas instituciones capaces de dotar a las comunidades y a los individuos que las componen de una nueva normatividad. Nuevos valores, una ética secular común, que contribuiría a la transformación de las relaciones sociales y que sustituiría a los que nos han sido inoculados a través de la construcción de un sentido común neoliberal. Unos valores que deberían suponer una ampliación o apertura del círculo del "nosotros", es decir, unos que tendrían que contribuir a generar vínculos de solidaridad y fraternidad más allá de las que se darían solamente entre los miembros de una comunidad (Arrieta et al, 2018: 14). No es necesario señalar, que comportamientos más solidarios entre las personas que forman parte de una comunidad no tienen por qué suponer automáticamente una mayor solidaridad respecto del resto personas que no pertenecen a la misma (Zubero, 2003). La búsqueda de la autonomía humana y la justicia social y ecológica no debe quedar enclaustrada dentro de las paredes de las comunidades locales.

Desde la performatividad de la acción, los comunes tienen el potencial de acometer esta compleja tarea. La praxis común tiene la capacidad de crear nuevas instituciones y transformar así los valores en los que se apoya un sistema social. Hablaríamos así, de una red de comunidades que interactuarían desde las lógicas del compartir y no desde las del intercambio mercantil. Pasaríamos de obtener un rendimiento por el plusvalor generado a través del valor de cambio obtenido de una producción excedentaria en una economía de mercado a reubicar el valor de uso de los bienes y la reciprocidad y la cooperación entre comunidades e individuos en el centro 
de las interacciones. Entre otras muchas finalidades, comunidades para el gobierno de la energía que no se guiarían por el ánimo de lucro y que tratarían de evitar acabar cooptados por las lógicas mercantiles.

\section{LA CRISTALIZACIÓN DE LO COMÚN EN EL ÁMBITO ENERGÉTICO}

Tal como se ha planteado hasta el momento, son los modos de autogobierno desarrollados en una comunidad para la gestión (generación, uso, distribución, cuidado, administración, consumo etc.) de un bien y los principios éticos que se defienden y promueven, lo que determina si nos encontramos ante una práctica común y en qué nivel se está dando. A partir de la caracterización de los comunes previamente realizada y de la observación de experiencias de nuestro entorno se han extraído los siguientes criterios para la identificación de iniciativas que responden tanto a la dimensión normativa como aplicada de lo común.

La dimensión normativa se refiere a los valores que señalan la meta social deseable de las prácticas comunes y que son fuente de toda conducta dentro de la comunidad. Valores de la ética secular común a alcanzar mediante reglas de comportamiento o supuestos de conducta comunes a cada miembro de la comunidad. De cada uno de los valores que configuran la dimensión normativa cuelgan los criterios relacionados con el carácter aplicado de los comunes. Se define así una normatividad de los comunes que se concreta, entre otros, en modos de gobierno, tecnologías empleadas, mecanismos para favorecer la igualdad, etc. específicos de cada práctica común.

A continuación, se presentan algunas de las iniciativas comunes existentes a nivel estatal que cumplen con estas condiciones y que por ende pueden ser consideradas parte de la praxis instituyente común. Los criterios normativos y aplicados anteriores son, de hecho, una herramienta que permite la identificación de prácticas que, más allá de sus divergencias (personalidad jurídica, actividades que desarrollan, escala en la que actúan, etc.) forman parte de un mismo continuum denominado como paradigma de lo común. Así, por ejemplo, no resulta complejo comprender que la Economía Social y Solidaria y el nuevo cooperativismo junto con las ecoaldeas o los partenariados público-ciudadanos puedan ser parte de este paradigma.

De hecho, las siguientes iniciativas son sólo una muestra de la constelación de los comunes de la energía. No son ni mucho menos todas las que hay. Como alternativa a las cinco grandes eléctricas, hay múltiples realidades que han ido emergiendo en las últimas décadas y ofrecen una vía para la transición ecosocial. Propuestas y proyectos que, desde diversas aproximaciones, pero con elementos en común, comienzan a dibujar un modelo energético ecológicamente más sustentable y socialmente más democrático. 
Figura I: Desglose de la dimensión normativa y aplicada de lo común

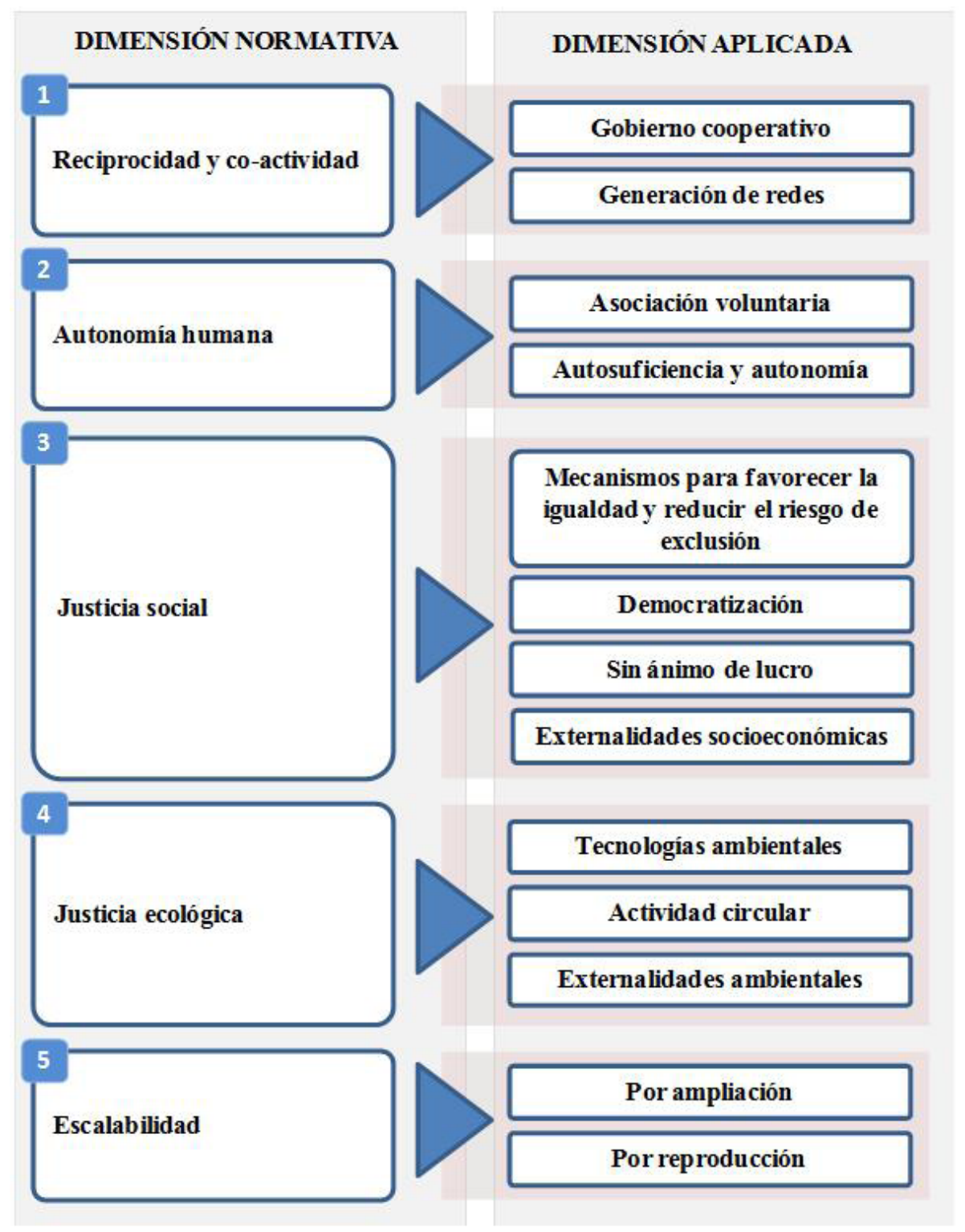

Fuente: elaboración propia. 
Tabla I: Comunidades autogestionarias

\begin{tabular}{|c|c|}
\hline Iniciativa & Descripción \\
\hline & $\begin{array}{l}\text { Instituto de Transición Rompe el Círculo } \\
\text { No sólo quieren promover una transición } \\
\text { como la que se está dando en muchos } \\
\text { lugares del mundo, sino que quieren hacerlo } \\
\text { dándole un enfoque anticapitalista, es decir, } \\
\text { experimentando con formas no capitalistas } \\
\text { de organización de la vida económica, social } \\
\text { y cultural. }\end{array}$ \\
\hline Arterra Bizimodu & $\begin{array}{l}\text { Arterra Bizimodu } \\
\text { Comunidad que aspira a ser un referente en } \\
\text { cuanto a la experimentación y la educación } \\
\text { hacia un modelo de desarrollo sostenible y } \\
\text { resiliente. }\end{array}$ \\
\hline $\begin{array}{l}\text { cardedeu } \\
\text { entransición }\end{array}$ & $\begin{array}{l}\text { Cardedeu en Transició } \\
\text { Formada por personas unidas por el } \\
\text { sentimiento de cooperación y comunidad, } \\
\text { tiene la intención de crear una red como } \\
\text { alternativa al sistema. Una comunidad } \\
\text { comprometida y activa para alcanzar una vida } \\
\text { de calidad y resiliente. }\end{array}$ \\
\hline Lakabe & $\begin{array}{l}\text { Lakabe } \\
\text { Un referente de neoruralismo, ocupación } \\
\text { rural, ecoaldeas, vida en comunidad, vida } \\
\text { ecológica. }\end{array}$ \\
\hline ASTRA & $\begin{array}{l}\text { Astra } \\
\text { Espacio autogobernado que incentiva la } \\
\text { participación directa de la ciudadanía y el } \\
\text { tejido social en la gestión de un espacio } \\
\text { público y comunitario. }\end{array}$ \\
\hline
\end{tabular}


Tabla II: Cooperativas de energía renovable ${ }^{11}$ y empresas sociales

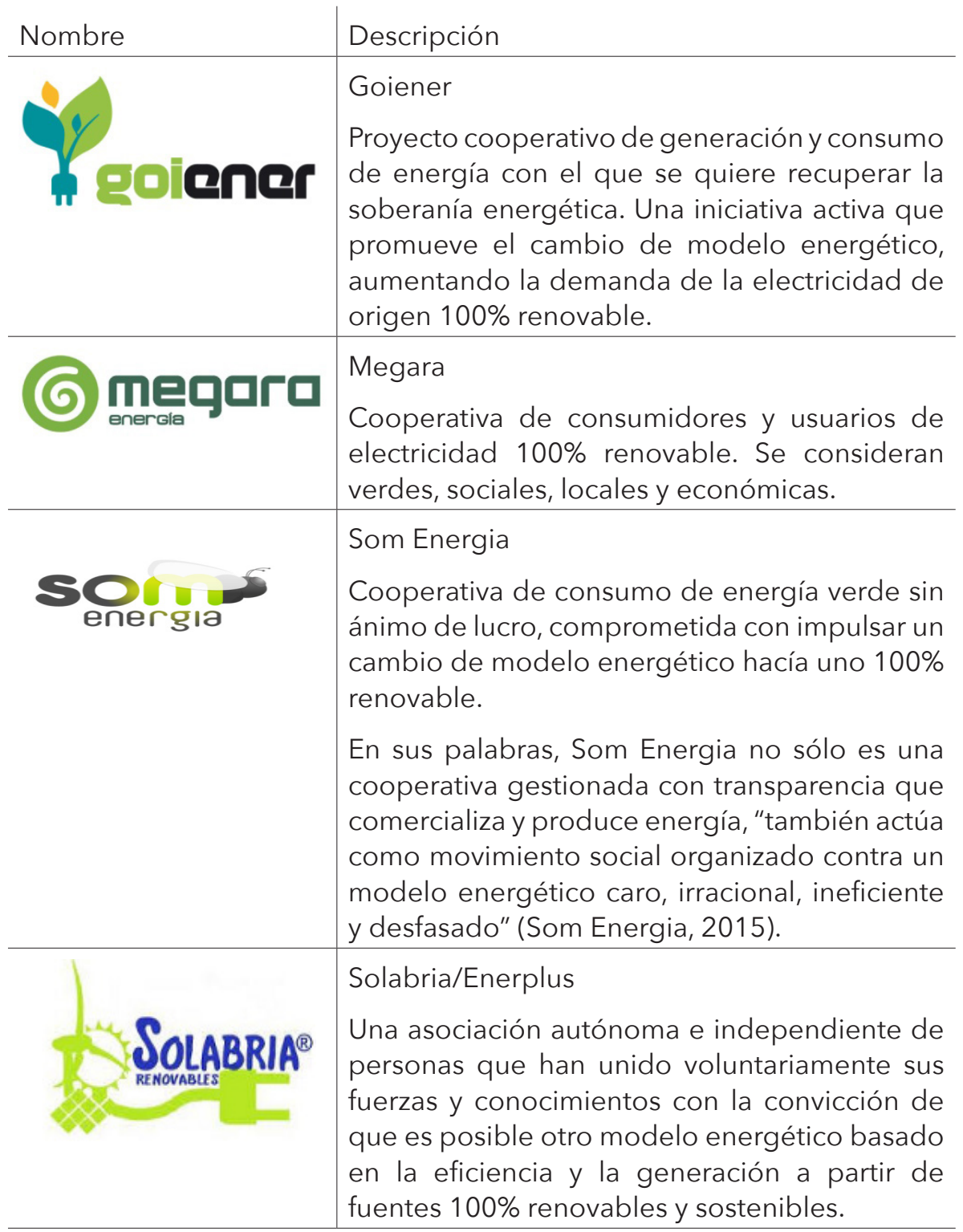

11 Al igual que ocurre con el resto de iniciativas, existen al menos otras 15 Cooperativas de Energía Renovable que operan actualmente a nivel estatal. Las que se presentan en están tablas son iniciativas estatales pertenecientes al paradigma de lo común que, cumpliendo con los criterios expuestos, han sido seleccionados de manera aleatoria como muestra de la constelación de iniciativas existentes. 


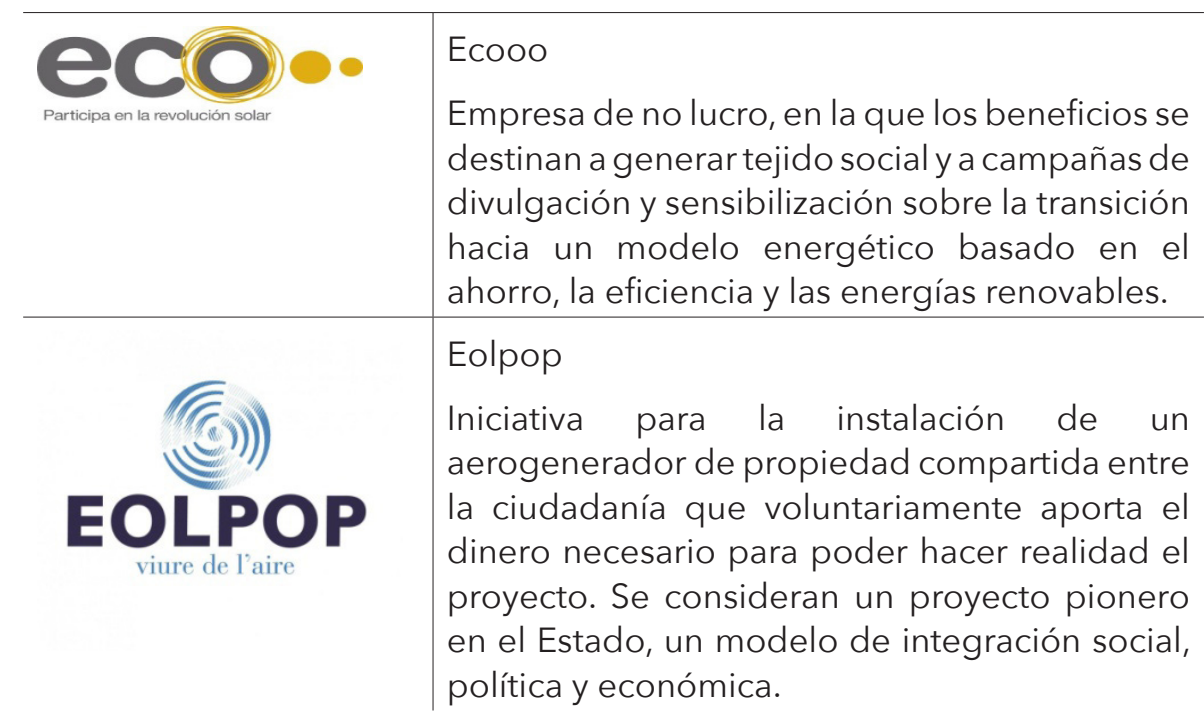

Tabla III: Partenariado público-ciudadano

\begin{tabular}{|l|l|}
\hline Nombre & Descripción \\
\hline & $\begin{array}{l}\text { Rubí Brilla } \\
\text { Uno de los proyectos estratégicos impulsado } \\
\text { desde el Ayuntamiento de Rubí cuyo objetivo } \\
\text { es que la ciudad se convierta en un referente } \\
\text { en la eficiencia energética y el uso de } \\
\text { energías renovables en entornos industriales, } \\
\text { comerciales y domésticos. } \\
\text { El objetivo a medio plazo es que la dirección } \\
\text { del proyecto sea mancomunada entre los } \\
\text { diversos grupos de interés que conforman } \\
\text { la ciudad (empresas, ciudadanos, etc.), } \\
\text { asumiendo como suyo Rubí Brilla e } \\
\text { implicándose en las decisiones de las } \\
\text { acciones a seguir. }\end{array}$ \\
\hline
\end{tabular}


Tabla IV: Investigación y observatorios especializados

\begin{tabular}{|l|l|}
\hline Nombre & Descripción \\
\hline Observatorio Crítico de la Energía \\
\hline & $\begin{array}{l}\text { Compuesto por un grupo de jóvenes } \\
\text { ingenieros/as y científicos que, unidos } \\
\text { en torno a un análisis común de los } \\
\text { problemas sociales y su creciente } \\
\text { gravedad, deciden comenzar una } \\
\text { actividad pública orientada a contribuir } \\
\text { a la mejora de la sociedad en asuntos } \\
\text { relacionados con la energía. }\end{array}$ \\
\hline
\end{tabular}

Fuente: elaboración propia.

Como parte de una realidad compleja, heterogénea y dispar, lo común, con todas las implicaciones prácticas que conlleva, se presenta en el ámbito de la energía en diferentes graduaciones, siendo cuanto menos difícil establecer los límites de lo que se podría considerar una praxis instituyente común de lo que no. Esta dificultad no sólo tiene su origen en los, muchas veces, frágiles intersticios existentes entre lo público, lo privado, lo social y lo común. La propia praxis, en su actividad diaria, puede no tener conciencia de estar generando nuevas condiciones, al mismo tiempo que "alteran el antiguo estado de cosas y hacen existir lo que no tiene ningún presente en la historia (Laval y Dardot, 2015: 495). Aquí se encuentra la complejidad, pero también el interés y el valor de estudiar una realidad que se encuentra en trepidante transformación. Comunes conscientes e inconscientes capaces de crear instituciones comunes.

\section{LA ALTERNATIVA ECOSOCIAL DESDE LO COMÚN}

Todo modelo que surge y se instala para la promoción de las bondades del sistema capitalista conlleva la defensa y el impulso de ciertos valores y hábitos socialmente y ecológicamente injustos e insostenibles. El perpetuo crecimiento del consumo y la producción requerido por el sistema actual se cimenta en un específico sentido común construido. La generalización de una alternativa sistémica necesita incidir en la transformación de estos valores y proponer otros alternativos. Desde la práctica, los comunes propician los comportamientos auto-contenidos y la suficiencia. Muestran que una vida buena y digna puede ser desacoplada del continuo crecimiento económico. En su dimensión local, la vinculación con el territorio en el que se activan favorece el reconocimiento de los límites físicos a los que la actividad humana está sujeta. Límites que hace tiempo han sido sobrepasados por los considerados países desarrollados. 
Hoy en día, las tecnologías posibilitan la generación y consumo distribuido de una parte de la energía que requerimos. Sin embargo, los avances tecnológicos, aunque puedan ser en ciertos casos necesarios, no son suficientes para que la transición ecosocial se produzca. La transformación a sociedades socialmente y ecológicamente más justas está condicionada por opciones políticas. De hecho, la descentralización de la producción no conlleva automáticamente una reapropiación ciudadana del modelo energético. La reapropiación y la distribución del poder requieren la socialización de los medios de producción. Para ello, los comunes, gracias a los modos de gobierno participativo y cooperativo que instauran y a la promoción que hacen de la autoproducción y el autoconsumo, podrían aprovecharse de manera óptima del potencial democratizador de estas tecnologías distribuidas.

Ante la racionalidad financiera propagada en la fase neoliberal, en parte también por las instituciones públicas, los comunes propician la asunción de valores como la reciprocidad y la solidaridad. El reconocimiento de la energía como bien social necesario para la autonomía humana y para la satisfacción de las necesidades más básicas, abre la posibilidad a una alternativa relación con la misma. Comunidades de comunidades que interactúan desde la solidaridad y el compartir y no desde la obtención de un rendimiento económico como finalidad última de los posibles intercambios. La inherente tendencia a la sobreproducción se vería en cierta medida atenuada al no ser el crecimiento económico el objetivo principal de la actividad humana.

Sin embargo, el autogobierno debe ir de la mano de nuevas instituciones capaces de dotar de una nueva normatividad. Una normatividad que suponga una apertura de las comunidades y no un ensimismamiento. La solidaridad y la reciprocidad no deben limitarse a los miembros de la comunidad, la praxis instituyente común debe tomar una posición abierta que, a través de la cooperación intercomunitaria, permita escalar hasta la consecución de mayores cotas de justicia social y ecológica a nivel global. El carácter local de los comunes, ya sea desde la generación de la energía como desde su consumo, implica una necesaria acción reticular para poder dar respuesta a problemáticas que han adquirido una escala global.

\section{BIBLIOGRAFÍA}

Alonso, L., Álvarez, I., Bellver, J. et al (2017).Rebeldías en común: Sobre comunales, nuevos comunes y economías cooperativas. Madrid: Ecologistas en Acción.

Arrieta et al (2018). La comunidad el rescate: algunas reflexiones sobre las políticas estatales de activación comunitaria en Europa. Zerbitzuan №65 (pp. 5-18). Donostia: SIIS Centro de Documentación y Estudios. 
Bauman, Z. (2015). Ceguera moral. La pérdida de sensibilidad en la modernidad líquida. Barcelona: Paidós.

Bollier, D. (2003).El redescubrimiento del procomún. Biblioweb. http://biblioweb.sindominio.net/telematica/bollier.html [consulta: 09/07/2013]. _(2009).Viral Spiral: How the Commoners Built a Digital Republic of Their Own. NuevaYork/Londres: The New Press.

Byrne, J., y Glover, L. (2002). A common future or towards a future commons: Globalization and sustainable development since UN. International Review for Environmental Strategies, Vol. 3, No. 1, 5-25.

Byrne, J. et al (2004). Reclaiming the atmospheric commons: Beyond Kyoto. Climate Change: Five Years after Kyoto. (pp. 429-452). Enfield: Science Publishers.

Castoriadis, C. (2007). La institución imaginaria de la sociedad, volumen 1: Marxismo y teoría revolucionaria, volumen 2: El imaginario social y la institución.Barcelona: Tusquets. ISBN 9788483834909

Consejo de la Unión Europea (2019).Proposal for a Directive of the European Parliament and of the Council (5076/19) on common rules for the internal market in electricity (recast) - Analysis of the final compromise text with a view to agreement.Bruselas: Consejo de la Unión Europea.https:// data.consilium.europa.eu/doc/document/ST-5076-2019-INIT/en/pdf [consulta: 25/10/2019].

Geiselberg, H. (ed.) (2017).The Great Regression. Cambridge: Polity Press.

Fukuyama, F. (1992). El Fin de la Historia y el Último Hombre. Barcelona: Planeta.

Fundación Energías Renovables (2011). Propuestas de Política Energética: el camino hacia un modelo energético sostenible. Madrid:

Fundación Energías Renovables (2013). Reforma energética: un proyecto de involución, Fundación Energías Renovables: Madrid: Fundación Energías Renovables http://fundacionrenovables.org/wp-content/ uploads/2013/09/La-ultima-reforma-FR-17-09-13.pdf[consulta: 08/08/2016].

Garcia, J. y Martín J.A. (2014). Seguridad Jurídica en Renovables: fundamentos para una estrategia procesal ante la reforma eléctrica, Madrid: Imedia comunicación.

Gómez, J.L. et al (2008). Historia Universal. New Jersey: Prentice Hall. Harvey, D. (2005). El "nuevo" imperialismo: acumulación por desposesión. Buenos Aires: CLACSO

Holloway, J. (2011). Agrietar el capitalismo: El hacer contra el trabajo. Barcelona: Intervención cultural.

Huerta, M.H. (2005). .El neoliberalismo y la conformación del Estado subsidiario. Política y Cultura No24 (pp. 121-150). Xochimilco: Universidad Autónoma Metropolitana. http://www.redalyc.org/articulo. oa?id=26702406[ consulta: 01/08/2016]. 
Klein, N. (2001). Reclaiming the commons. NewLeftReview N9. mayojuniohttp://newleftreview.org/ll/9/naomi-klein-reclaiming-the-commons [consulta: 02/02/2018].

Lafuente, A. (2007). El carnaval de la tecnociencia. Madrid: Gadir.

Laval C. y Dardot P. (2015). Común. Ensayo sobre la revolución en el siglo XXI. Barcelona: Gedisa.

Lilla, M. (2016).The Shipwrecked Mind: On Political Reaction. Nueva York: The New York Review of Books.

Linebaugh, P. (2010). Some Principles of the Commons. onthecommons. org. Minneapolis: Commons Magazine. http://onthecommons.org/ some-principles-commons[consulta: 18/07/2013].

Martil, I. (2016). Los privilegios del oligopolio eléctrico. Público. http://blogs. publico.es/econonuestra/2016/01/09/los-privilegios-del-oligopolioelectrico/ [consulta: 29/07/2016].

Martínez-Alier, J. y Oliveras, A. (2003). ¿Quién debe a quién? Deuda ecológica y Deuda Externa. Barcelona: Icaria.

Martinez, L. (2014). Productores de nuestra propia energía. Plantas colectivas y autoconsumo.Alta Tensión: por un nuevo modelo energético sostenible, democrático y ciudadano. Barcelona: Icaria.

Mishra, P. (2017).Age of Anger: A History of the Present. Reino Unido: Penguin Books.

Mitchell, T. (2011). Carbon Democracy political power in the age of oil. Londres: Verso.

National Footprint Accounts (2016). Ecological Wealth of Nations. Global Footprint Network. http://www.footprintnetwork.org/ecological_ footprint_nations/ [consulta: 28/02/2018].

Ostrom, E. (2000).El Gobierno de los Bienes Comunes. La Evolución de las Instituciones Colectivas. Ciudad de México: Fondo de Cultura Económica de México.

Petrella, R. (2009). Una narración de la historia: Compartir bienes, vivir en común. Barcelona: Intermón Oxfam.

Pradel, M. et al (2018). El momento de la ciudadanía: innovación social y gobernanza urbana. Madrid: Catarata.

Ramonet, Ignacio (2011). El nuevo sistema-mundo. Le Monde Diplomatique $\quad \mathrm{N}^{\circ} \quad 192$. http://www.monde-diplomatique. es/?url=editorial/0000856412872168186811102294251000/ editorial/?articulo=13b9ad43-3237-4ad5-b889-e064cc43ac62 [consulta: 25/10/2016].

Rayner, T. (2012). Commoning is making common. Philosophy for change. Enmore.http://philosophyforchange.wordpress.com/2012/03/08/ commoning/[consulta: 18/07/2013]. 
Rendueles, C. (2017). Las condiciones institucionales de una reconstrucción de los bienes comunes en sociedades mercantilizadas. Rebeldías en común: Sobre comunales, nuevos comunes y economías cooperativas. Madrid: Ecologistas en acción.

Riechman, J. (2004). Gente que no quiere viajar a marte: ensayo sobre ecología, ética y autolimitación. Madrid: Catarata.

Riutort, S. (2017). Energia para la democracia. Madrid: Fuhem Ecosocial.

Rostow, W. W. (1961). Las etapas del crecimiento económico. México: Fondo de Cultura Económica.

Sacristán, M. (2003). M.A.R.X. Máximas, aforismos y reflexiones con algunas variables libres. Barcelona: El viejo topo.

Streeck, W. (2017). ¿Cómo terminará el capitalismo? Ensayos sobre un sistema en decadencia. Madrid: Traficantes de sueños.

Taibo, C. (2011). El decrecimiento explicado con sencillez. Madrid: Catarata.

Tirado, S., et al (2014). Pobreza energética en España. Análisis de tendencias. Madrid: Asociación de Ciencias Ambientales.

Urkidi, L. et al (2015). Transiciones energéticas: sostenibilidad y democracia energética. Bilbao: Universidad del País Vasco/Euskal Herriko Uniberstitatea.

Zubero, I. (2003). El reto de la inmigración acoger al otro y ampliar el nosotros. Ciudadanía, multiculturalidad e inmigración (pp. 135-164). Iruña: Verbo Divino. ISBN 848169567X. 